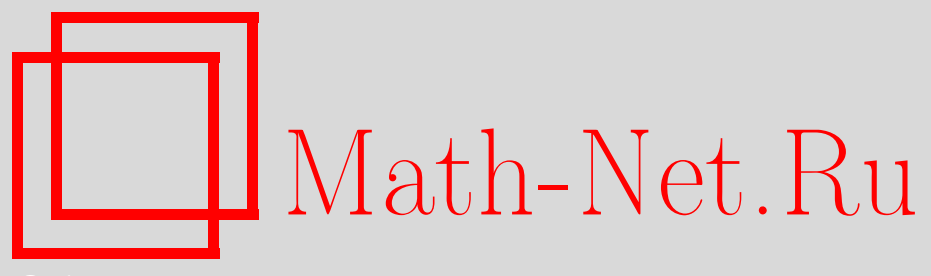

С. А. Теляковский, О коэффициентах рядов Фурье, сходящихся в L, Матем. заметки, 2015, том 98, выпуск 1, 156-157

DOI: https://doi.org/10.4213/mzm10695

Использование Общероссийского математического портала Math-Net.Ru подразумевает, что вы прочитали и согласны с пользовательским соглашением http://www . mathnet.ru/rus/agreement

Параметры загрузки:

IP : 54.81 .137 .203

26 апреля 2023 г., 14:12:17

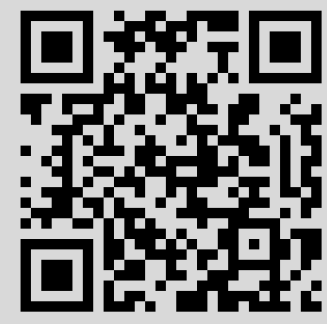




\section{О коэффициентах рядов Фурье, сходящихся в $L$}

\section{С. А. Теляковский}

В настоящей заметке усиливается результат Фомина [1; теорема 3], согласно которому, если ряд Фурье по косинусам

$$
\frac{a_{0}}{2}+\sum_{k=1}^{\infty} a_{k} \cos k x
$$

сходится в метрике $L$, то для каждой последовательности натуральных чисел $\left\{s_{n}\right\}$ такой, что $s_{n} \leqslant n$,

$$
\lim _{n \rightarrow \infty} \sum_{k=1}^{s_{n}} \frac{1}{k} a_{n+k}=0
$$

Пусть $\Lambda$ - строго возрастающая последовательность натуральных чисел $\left\{n_{j}\right\}, n_{1}=1$. В [2; теорема 1] установлено, что если для последовательности $\Lambda$ существует такое число $M$, что при всех $i$

$$
\sum_{j=i}^{\infty} \frac{1}{n_{j}} \min \left(n_{i}, n_{j+1}-n_{j}+1\right)<M,
$$

то справедлива оценка

$$
\sum_{j=1}^{\infty}\left|\sum_{l=n_{j}}^{n_{j+1}-1} \frac{\sin l x}{l}\right| \leqslant 3+M .
$$

Для коэффициентов ряда (1) определим числа

$$
a_{k}(n)= \begin{cases}a_{k}, & k=n+1, \ldots, 2 n, \\ 0 & \text { при остальных } k\end{cases}
$$

Теорема. Если ряд (1) является рядом Фуръе, сходящимся в метрике L, и для последовательности $\Lambda$ выполнено условие (2), то при $n \rightarrow \infty$

$$
\sum_{j=1}^{\infty}\left|\sum_{l=n_{j}}^{n_{j+1}-1} \frac{a_{n+k}(n)}{k}\right| \rightarrow 0 .
$$

ДокАЗАТЕльство. Из условия теоремы следует, что значения интегралов

$$
\int_{0}^{\pi}\left|\sum_{k=1}^{\infty} a_{n+k}(n) \cos (n+k) x\right| d x
$$

стремятся к нулю при $n \rightarrow \infty$.

Пользуясь оценкой (3), находим

$$
\sum_{j=1}^{\infty}\left|2 \sin n x \sum_{l=n_{j}}^{n_{j+1}-1} \frac{\sin l x}{l}\right|=\sum_{j=1}^{\infty}\left|\sum_{l=n_{j}}^{n_{j+1}-1} \frac{1}{l}[\cos (n-l) x-\cos (n+j) x]\right| \leqslant 6+2 M .
$$

Исследование выполнено за счет гранта Российского научного фонда (проект 14-50-00005).

(C) С. А. ТЕляковский, 2015 
Следовательно, при $n \rightarrow \infty$ стремятся к нулю величины

$$
\begin{aligned}
& \int_{0}^{\pi} \sum_{j=1}^{\infty}\left|\sum_{l=n_{j}}^{n_{j+1}-1} \frac{1}{l}[\cos (n-l) x-\cos (n+l) x] \sum_{k=1}^{n} a_{n+k}(n) \cos (n+k) x\right| d x \\
& \geqslant \sum_{j=1}^{\infty}\left|\int_{0}^{\pi} \sum_{l=n_{j}}^{n_{j+1}-1} \frac{1}{l}[\cos (n-l) x-\cos (n+l) x] \sum_{k=1}^{n} a_{n+k}(n) \cos (n+k) x d x\right| \\
& =2 \sum_{j=1}^{\infty}\left|\sum_{k=n_{j}}^{n_{j+1}-1} \frac{1}{k} a_{n+k}(n)\right| .
\end{aligned}
$$

Теорема доказана.

Отметим, что если ослабить условие на ряд (1) и предполагать не его сходимость в метрике $L$, а только равномерную ограниченность норм в $L$ частных сумм этого ряда, то аналогичные рассуждения покажут равномерную ограниченность величин из левой части (4).

Точно так же устанавливается справедливость подобных утверждений для рядов по синусам. При этом вместо (5) используется равномерная ограниченность сумм рядов

$$
\sum_{j=1}^{\infty}\left|\cos n x \sum_{l=n_{j}}^{n_{j+1}-1} \frac{\sin l x}{l}\right| .
$$

\section{СПИСОК ЦИТИРОВАННОЙ ЛИТЕРАТУРЫ}

[1] Г. А. Фомин, Матем. сб., 110:2 (1979), 251-265. [2] А. С. Белов, С. А. Теляковский, Матем. сб., 198:6 (2007), 25-40.

\section{С. А. Теляковский}

Математический институт им. В. А. Стеклова РАН

E-mail: sergeyaltel@ya.ru
Поступило

12.01 .2015

Исправленный вариант

29.01 .2015 